\title{
Numerical Simulation of Ti-Based Metallic Glasses as Whipple Shield Bumper by Smoothed Particle Hydrodynamics Methods
}

\author{
Weiqi Tang 1,3,a, Kun Zhang 1,3,b*, Yansen Li ${ }^{1,3, c}$, Yang Wang ${ }^{1,3, d}$, \\ Yating Zhang ${ }^{1,3, f}$, Bingchen Wei i $^{1,2,3, \text { q* }^{*}}$
}

${ }^{1}$ Key Laboratory of Microgravity (National Microgravity Laboratory), Chinese Academy of Sciences, Institute of Mechanics, Beijing 100190, China

${ }^{2}$ Center of Materials Science and Optoelectronics Engineering, University of Chinese Academy of Sciences, Beijing 100049, China

${ }^{3}$ School of Engineering Science, University of Chinese Academy of Sciences, Beijing 100049, China

atangweiqi@imech.ac.cn, bzhangkun@imech.ac.cn, cliyansen@imech.ac.cn, dwangyang@imech.ac.cn, e'zhangyating@imech.ac.cn, 'weibc@imech.ac.cn

\begin{abstract}
Keywords: Metallic glasses (MGs), Hypervelocity impact, Whipple shield, Smoothed particle
\end{abstract} hydrodynamics (SPH)

Abstract. The debris clouds produced by hypervelocity impacts on Ti-based metallic glasses (Ti-MGs) and Al-6061-T6 bumper were studied by smoothed particle hydrodynamics (SPH) methods. The change of the vanguard shape, dispersion and ejection angle were also obtained with the same bumper thickness to the projectile-diameter ratio $(\mathrm{h} / \mathrm{d})$. For the same $\mathrm{h} / \mathrm{d}$ valve, the debris cloud of Ti-MGs bumper had a more widely dispersion and ejection angle than with Al-6061-T6 bumper; the vanguard velocity of Ti-MGs bumper was also lower than A1-6061-T6 bumper. Moreover, for the same bumper areal density, the vanguard of the debris cloud in MGs bumper was plane-shaped. This study demonstrates that Ti-MGs exhibit an excellent bumper protection performance, which asset can pave new paths for their further applications.

\section{Introduction}

The protection of spacecraft against micrometeoroids and human-made orbital debris is of considerable interest within the past decade. One of the most common ways which is called "Whipple shields" is effective at vaporizing and diffusing the debris associated with small projectile impacts [1]. A classical Whipple-type shield is comprised of two layers separated by a gap, called standoff. The outer layer, termed "bumper", is usually comprised of a thin panel of alloys or ceramics, while the back layer is usually the wall of the spacecraft. Whipple shields operate effectively against projectile impacts because the bumper can break up the projectile into debris cloud with the reduction of the loading intensity at the rear wall.

Aluminum alloys have been widely used in spacecraft and satellites as a shielding material for decades owing to their lightweight. For example, Al-6061-T6, comprised by $\mathrm{Si}, \mathrm{Mg}$, and $\mathrm{Cu}$, is commonly used as the construction of the bumper in the Whipple shields. However, the protective performance of aluminum alloy is still limited because of its low strength and impact toughness.

Compared with $\mathrm{Al}$ alloys, metallic glasses (MGs) have been a popular subject of research within the past decade, due to their unique mechanical and chemical properties, including high specific strength and good corrosion resistance [2-6]. They also exhibit excellent carbide-like hardness and fracture toughness making them ideal candidates for applications in the impact of aircraft and armor design [2,3], and bullet ballistics in forensic science [4]. Previous studies show that the Whipple shields integrated with layers of MGs exhibit a better hypervelocity impact performance than the one similar to what is used on the International Space Station [5]. The morphologies of the bullet holes exhibit three different regions: melting area, vein-pattern area, and radiating core feature area, suggesting that various regions experience different stress states during the hypervelocity impact. The damage features of MGs caused by hypervelocity impact were also investigated, in which the 
radially symmetric cracks form on the target surface, shear bands/cracks that are parallel to each other appear on the cross-section close to the shocking surface of MGs [6]. However, the experimental studies are known to offer only a limited scope of aftereffect information in this regard. It is difficult to analyze dynamic deformation process real-timely because of characteristics of the hypervelocity loadings and limitation of temporal-spatial resolution.

The smoothed particle hydrodynamics (SPH) [7-9] simulation is based on grid-less Lagrangian methods (coordinates are moving together with bodies), in which bodies are divided into discrete elements (particles) with spatial distances and their properties are smoothed out by a kernel function. The SPH method is significantly better than conventional grid methods without considering the advection or the grid recalculation. Moreover, the formation and development of ruptures in materials are also considered. This method has been proved to be efficient for solving the hypervelocity impact problem.

In the present work, the dynamic response of hypervelocity impact on the MGs bumper has been studied by SPH simulation. The bumper materials are Ti-based MGs, which have exceptional thermal stability, relatively low density, and very high specific strength. Al-6061-T6 alloys are also studied for comparison.

\section{Numerical Simulation}

SPH/FEM Model. The numerical simulations were performed using the smoothed particle hydrodynamics method (SPH) and finite element method (FEM) implemented in explicit software ANSYS/LS-DYNA. The numerical model is comprised of a $\mathrm{d}=5 \mathrm{~mm}$ sphere projectile made of Al-1100 and a $\mathrm{h}=1 \mathrm{~mm}$ bumper made of Ti-MGs. The projectile and the impact zone of the bumper, with a diameter of $10 \mathrm{~mm}$ which is bigger enough so that the boundary conditions don't influence the impact results, are set in the form of SPH elements; the other parts of bumper is set in the form of FEM elements. The bumper was divided into 8 particles to its thickness, and the sphere projectile divided into 40 particles to its diameter, that the diameter of one particle is $0.125 \mathrm{~mm}$.

In this simulation, we employed Johnson-Cook constitutive and fracture model [10, 11], Mie-Gruneison equation of state for projectile and bumper material. The Johnson-Cook constitutive model was selected because it allows taking into account a plastic strain rate and a temperature softening including a phase transition. It is important to consider it because projectile and plate materials melting and evaporation processes can occur at high velocities impact. Johnson-Cook model contains two parts consists of the constitutive model and the failure model.

The flow stress of ductile materials can be effectively quantified as:

$$
\sigma_{y}=\left(\mathrm{A}+\mathrm{B} \varepsilon_{p}^{n}\right)\left(1+\mathrm{Cln} \dot{\varepsilon}^{*}\right)\left(1-T^{*^{m}}\right)
$$

Where $\varepsilon_{p}$ is the equivalent plastic strain, $\dot{\varepsilon}^{*}=\dot{\varepsilon} / \dot{\varepsilon}_{0}$ is the dimensionless plastic strain rate for $\dot{\varepsilon}_{0}=1.0 \mathrm{~s}^{-1}$, and $T^{*}=\left(T-T_{r}\right) /\left(T_{m}-T_{r}\right)$ is the homologous temperature, $T_{m}$ and $T_{r}$ are melting temperature and room temperature, respectively. The five material constants are $A, B, n, C$ and $m$. In the current simulation, the room temperature, $T_{r}$ is $293 \mathrm{~K}$. The reference strain rate $\dot{\varepsilon}_{0}$ is simply taken as $1 \mathrm{~s}^{-1}$.

Moreover, the general expression for the strain at fracture is given by

$$
\varepsilon_{f}=\left(D_{1}+D_{2} \exp D_{3} \sigma^{*}\right)\left(1+D_{4} \ln \dot{\varepsilon}^{*}\right)\left(1+D_{5} T^{*}\right)
$$

Where $\sigma^{*}$ is the ratio of pressure divided by effective stress

$$
\sigma^{*}=\frac{\sigma_{m}}{\bar{\sigma}}
$$

Where the $\sigma_{m}$ is the average of the three normal stresses and $\bar{\sigma}$ is the von-Mises equivalent stress.

Previous studies about the Johnson-Cook constitutive model and Mie-Gruneisen equations of MGs have been carried out [12-18]. We chose the parameters from these works and fitted the Johnson-Cook fracture model parameter of MGs from these experiments data $[19,20]$ by least 
squares method. The material parameters are listed in the Tables 1-2. For comparison, we calculated 3 cases of different bumpers by this model (see Table 3 )

Table 1 Johnson-Cook model parameters for Ti-MGs, Al-1100, and Al-6061-T6 [21].

\begin{tabular}{lllll}
\hline Parameter & Unit. & Al-1100 & Ti-MGs & Al-6061-T6 \\
\hline$\rho$ & $\mathrm{kg} / \mathrm{m}^{3}$ & 2770 & 6000 & 2750 \\
$G$ & $\mathrm{~Pa}$ & $25.9 \mathrm{e} 9$ & $35.5 \mathrm{e} 9$ & $25 \mathrm{e} 9$ \\
$A$ & $\mathrm{~Pa}$ & $4.1 \mathrm{e} 7$ & $1.63 \mathrm{e} 9$ & $3.241 \mathrm{e} 8$ \\
$B$ & $\mathrm{~Pa}$ & $1.25 \mathrm{e} 8$ & $2.69 \mathrm{e} 8$ & $1.138 \mathrm{e} 8$ \\
$n$ & & 0.183 & 0.27 & 0.42 \\
$c$ & & 0.001 & 0.003 & 0.002 \\
$m$ & & 0.859 & 1.34 & 1.34 \\
Testing temperature & $\mathrm{K}$ & 293 & 293 & 293 \\
Melting temperature & $\mathrm{K}$ & 893 & 1425 & 893 \\
Testing strain rate & $\mathrm{s}-1$ & 1 & 1 & 1 \\
Specific heat capacity, $C_{p}$ & $\mathrm{~J} /(\mathrm{kg} \cdot \mathrm{K})$ & 910 & 830 & 910 \\
Coefficients in the Johnson-Cook failure model & & & \\
$D_{1}$ & & 0.071 & 0.013 & -0.77 \\
$D_{2}$ & & 1.248 & 0.0031 & 1.45 \\
$D_{3}$ & & -1.142 & -2.263 & -0.47 \\
$D_{4}$ & & 0.0097 & -0.00256 & 0 \\
$D_{5}$ & & 0 & 1.6 \\
\hline & & & & \\
\hline
\end{tabular}

Table 2 Mie-Gruneisen equation of state parameters coefficients for Ti-MGs and Al-1100.

\begin{tabular}{lllllllll}
\hline & $C_{0, \mathrm{~m} / \mathrm{s}}$ & $S_{1}$ & $S_{2}$ & $S_{3}$ & $a$ & $E_{0}$ & $\Gamma_{0}$ & $V_{0}$ \\
\hline Ti-MGs & 4241 & 1.015 & 0 & 0 & 0 & 0 & 1 & 1 \\
Al-1100 & 3935 & 1.578 & 0 & 0 & 0 & 0 & 1.69 & 1 \\
Al-6061-T6 & 5240 & 1.4 & 0 & 0 & 0 & 0 & 1.97 & 1 \\
\hline
\end{tabular}

Table 3 Different cases configurations.

\begin{tabular}{llllll}
\hline Case & Bumper material & $\mathrm{d}(\mathrm{mm})$ & $\mathrm{V}_{\mathrm{i}}(\mathrm{km} / \mathrm{s})$ & $\mathrm{h}(\mathrm{mm})$ & $\mathrm{V}_{\mathrm{r}}(\mathrm{km} / \mathrm{s})$ \\
\hline 1 & Ti-MGs & 5 & 6 & 1 & 4.97 \\
2 & Al-6061-T6 & 5 & 6 & 1 & 5.42 \\
3 & Ti-MGs & 5 & 6 & 0.5 & 5.33 \\
\hline
\end{tabular}

Simulation Results and Discussion. When the hypervelocity particles impact on the bumper, a debris cloud appears which is comprised mostly of bumper fragments emanating behind the bumper. Fig. 1 shows the morphology of the debris cloud produced by a $5 \mathrm{~mm}$ diameter Al-1100 sphere impacting a $1 \mathrm{~mm}$ thick Ti-MGs bumper and Al-6061-T6 bumper respectively. As seen in Fig. 1, the 
debris clouds formed by the impact of the Al-1100 projectile with Ti-MGs bumper and Al-6061-T6 bumper produce a similar debris-cloud structure. The bubbles of bumper debris mainly trail and surround the major concentration of projectile debris. There is the internal structure of projectile debris located contiguous behind the front of the bubble of bumper debris. A majority of the debris cloud mass is concentrated in the internal structure of projectile debris. Fig. 2 illustrates the quantity distribution of the SPH element behind the bumpers which can represent the debris distribution. For the $\mathrm{x}$-direction and y-direction, the debris with Ti-MGs bumper seems more loosely distributed in the entire debris cloud comparing with Al-6061-T6 bumper.

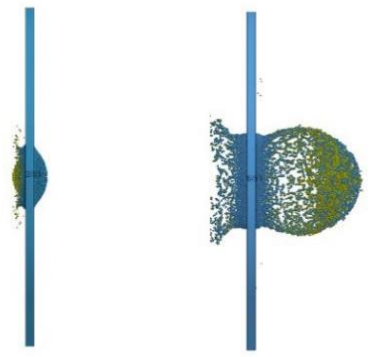

0.6 us

3 us

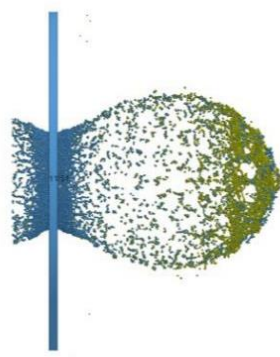

6 us

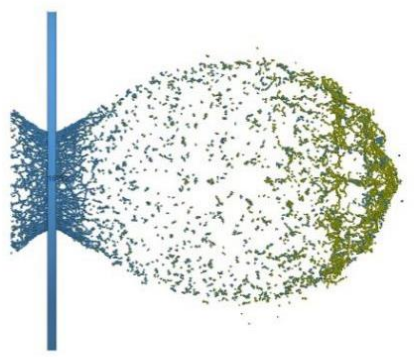

9 us

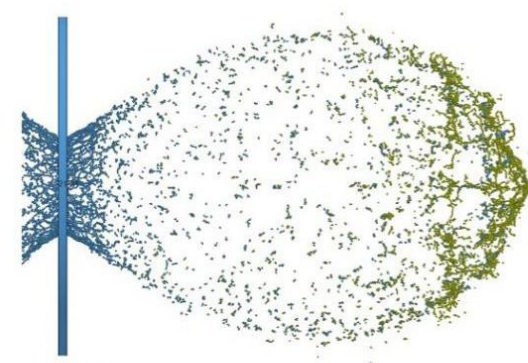

12 us

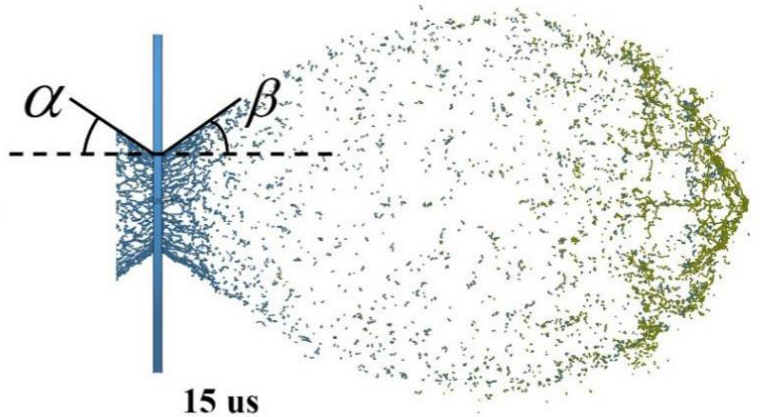

(a) Ti-MGs bumper

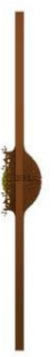

0.6 us

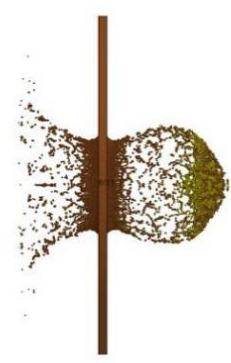

3 us

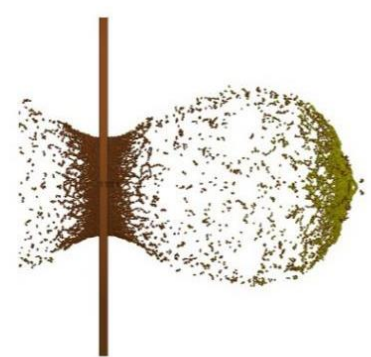

6 us

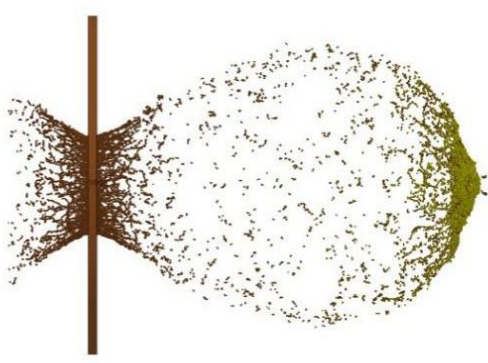

9 us

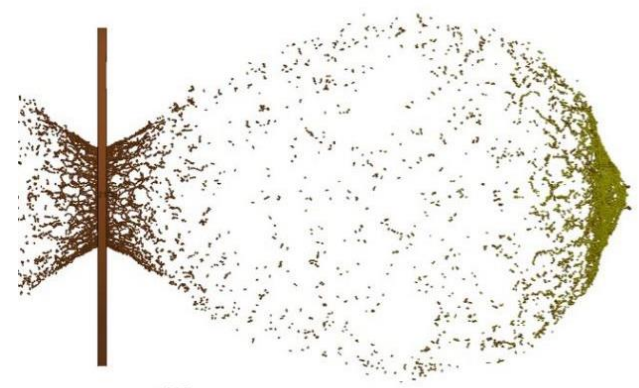

12 us

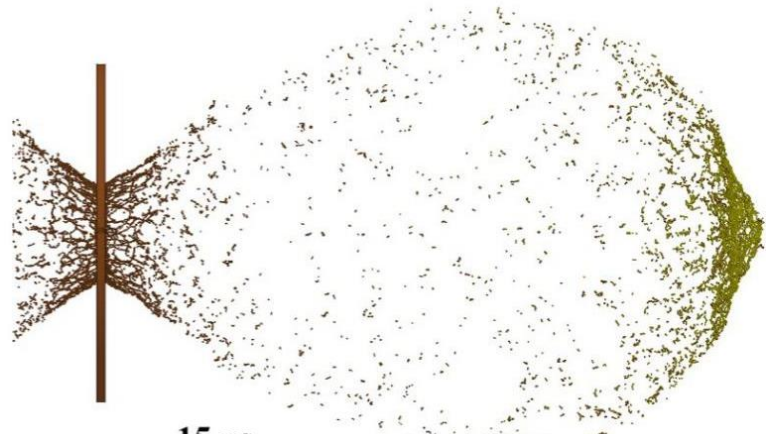

15 us

(b) Al-6061-T6 bumper

Fig. 1 The evolution of debris cloud produced by the impact of Al-1100 sphere projectile at a velocity of $6 \mathrm{~km} / \mathrm{s}$ : (a) Ti-MGs bumper and (b) Al-6061-T6 bumper. 
Unlike the vanguard of the debris cloud of $\mathrm{Al} / \mathrm{Al}$, the vanguard of the $\mathrm{Al} / \mathrm{MGs}$ debris cloud appears delamination, which makes the mass of debris cloud not concentrated in the front of the internal structure. The vanguard velocity of the front in the debris cloud is obtained as shown in Fig. 3 and Table 3. It is noted that the maximum vanguard velocity of $\mathrm{Al} / \mathrm{Al}$ debris cloud is nearly $5.42 \mathrm{~km} / \mathrm{s}$ while the maximum vanguard velocity of A1/MGs debris cloud is just about $4.97 \mathrm{~km} / \mathrm{s}$. That means the Ti-MGs exhibit better bumper protection performance than Al-6061-T6 alloys.

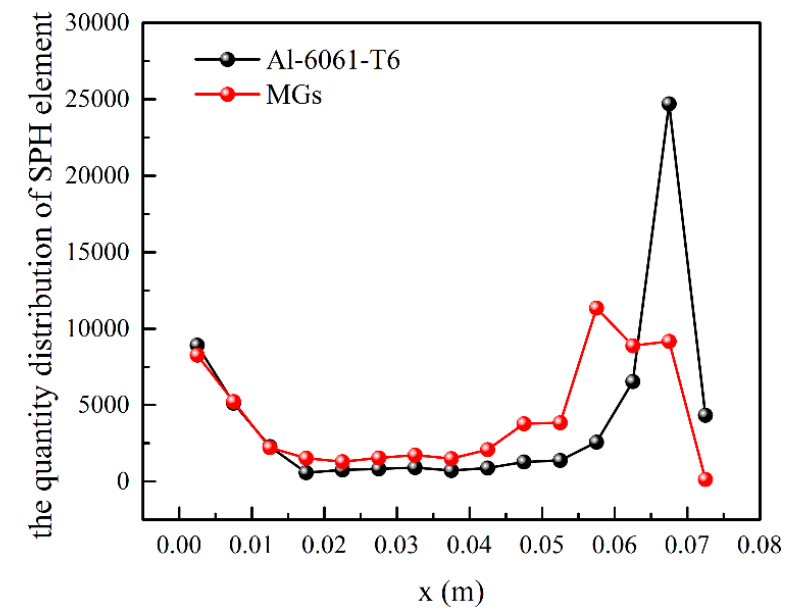

(a)

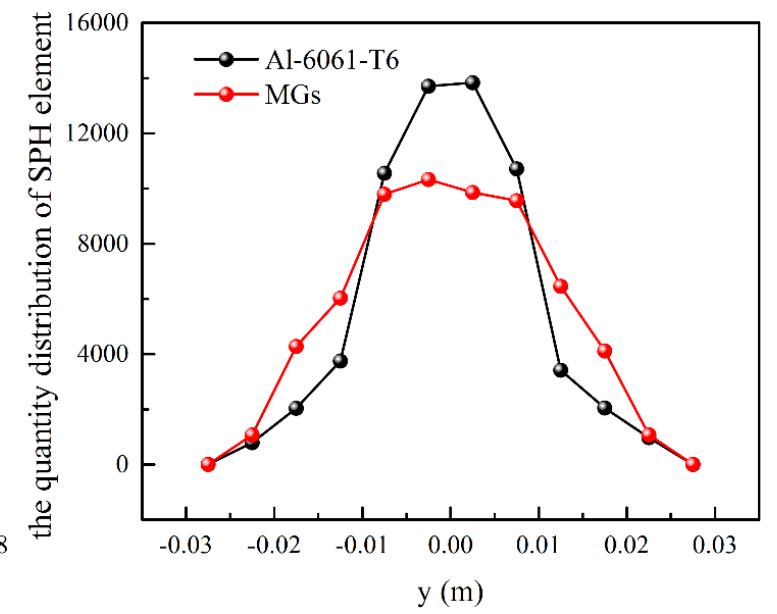

(b)

Fig. 2 The quantity distribution of SPH element behind the bumpers. (a) x-direction distribution and (b) y-direction distribution.

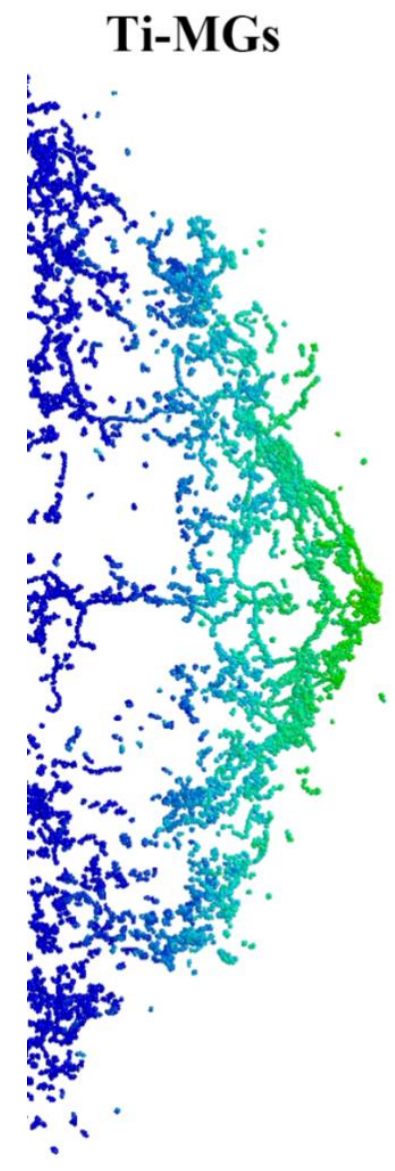

(a)

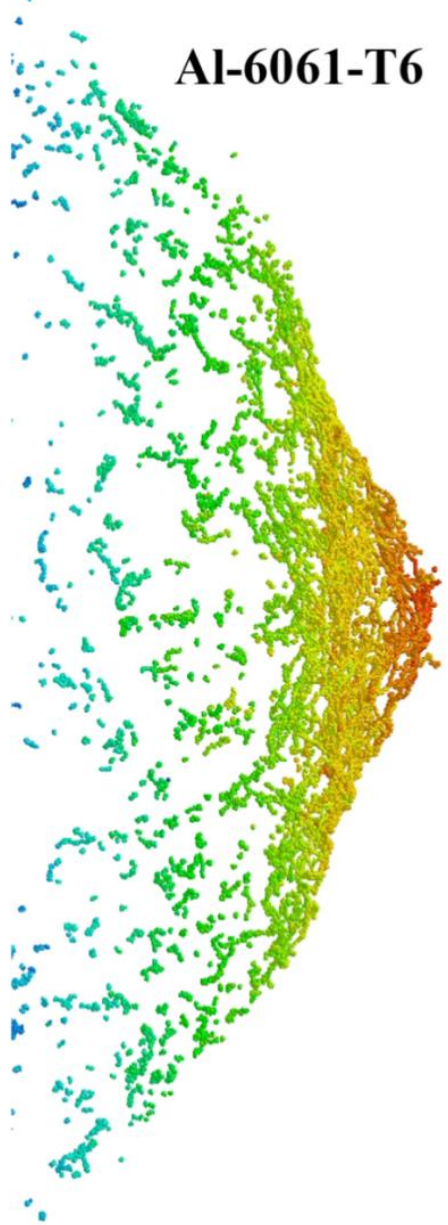

(b)

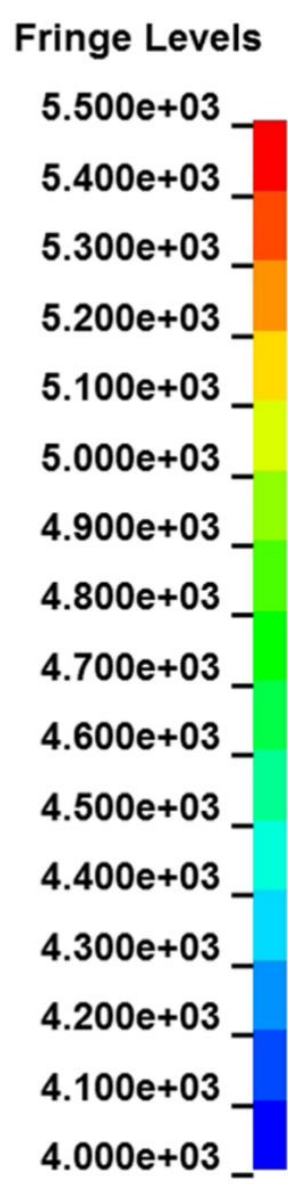

Fig. 3 The contour of the vanguard velocity of (a) Ti-MGs and (b) Al-6061-T6 alloys. 
On the other hand, the ejection and dispersion angles of the debris cloud, which are formed left and right the bumper during hypervelocity impact, are also the effective parameters for evaluating the bumper protection. Fig. 4 shows the change of the ejection and dispersion angle during the impact. Both two angles have a similar trend and can be described as two stages: firstly, the impact hole is expanding and tensile deformation occurs at the hole edge, which is resulted that the ejection and dispersion angles are smaller than 0 degrees; next, when the impact hole stops expanding, the bumper materials at the hole edge are fractured and the debris spread in the direction of velocity. With smaller elongation at rupture, impact holes at MGs bumper stops expanding more prematurely than Al-6061-T6 bumper. Moreover, both angles of the MGs bumper are larger than Al-6061-T6 bumper, which means that the debris cloud can spread more dispersive.

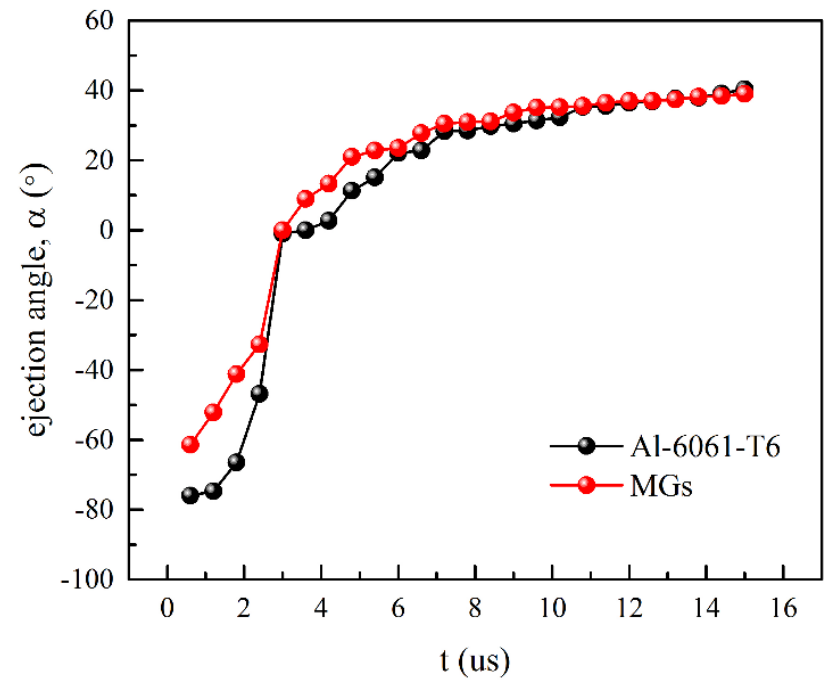

(a)

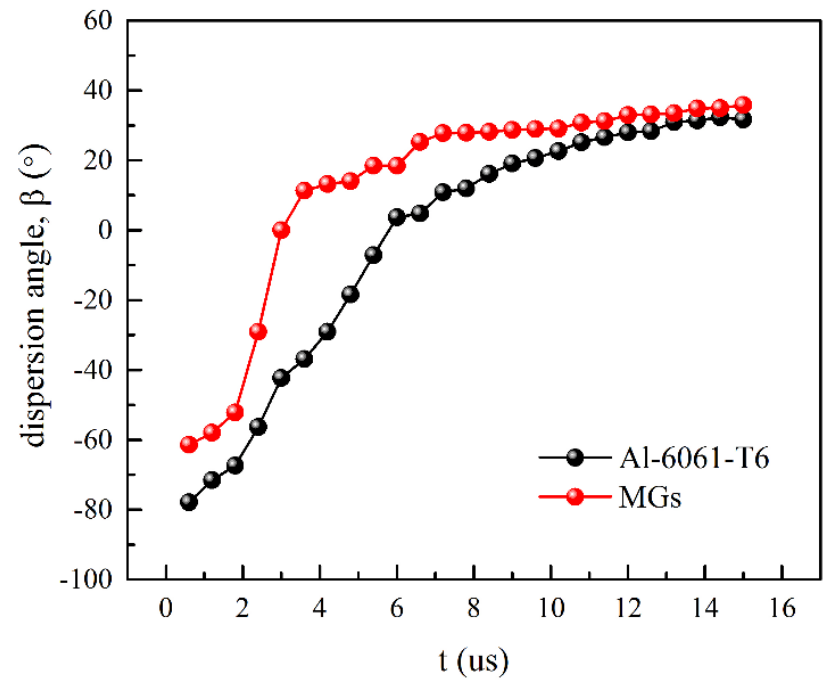

(b)

Fig. 4 The change of the ejection and dispersion angle with time. (a) ejection angle and (b) dispersion angle.

To explain the different effects between using Ti-MGs bumper and Al-6061-T6 bumper in the Whipple shields, the mechanical response of sampling points along with the bumper thickness in the central impact zone is obtained. The curves of different mechanical parameters (pressure, stress, strain) are shown in Fig. 5. Fig. 5a,b show that the pressure has experienced a sharp rise and then decreases dramatically when reaching the maximum at about 0.1-0.2 us after impact. During the penetration, the energy is continuously absorbed or transmitted, which lead to a decrease in both the peak value and width of the pressure pulse along with the bumper thickness. Comparing the sampling points at the same position, the peak value of pressure in the MGs bumper is larger than the Al-6061-T6 bumper all the time. This may be one of the reasons that the MGs has a more effective ability that breaks the projectile and absorbs the kinetic energy.

Moreover, Fig. 5c,d show that the plastic strain of sampling points at the same position with Ti-MGs bumper is slightly lower than with Al-6061-T6 bumper. Compared to the fracture strain under the uniaxial compression from the previous work [22, 23], the Ti-MGs is more brittle than Al-6061-T6. While at the ultrahigh strain rate, the fracture strain difference between Ti-MGs and Al-6061-T6 is not significant than at the quasi-static condition. Furthermore, as shown in Fig. 5e,f, the peak of von-Mises stress in Ti-MGs is nearly $1800 \mathrm{MPa}$ which far greater than $350 \mathrm{MPa}$ in A1-6061-T6 alloy because Ti-MGs have greater strength to density ratio than A1-6061-T6 alloy. 
Ti-MGs

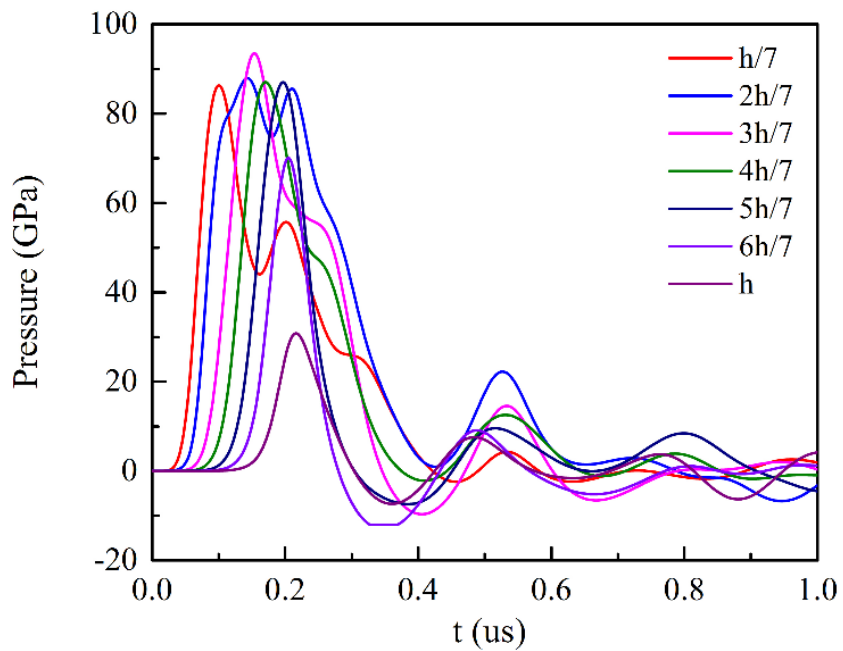

(a)

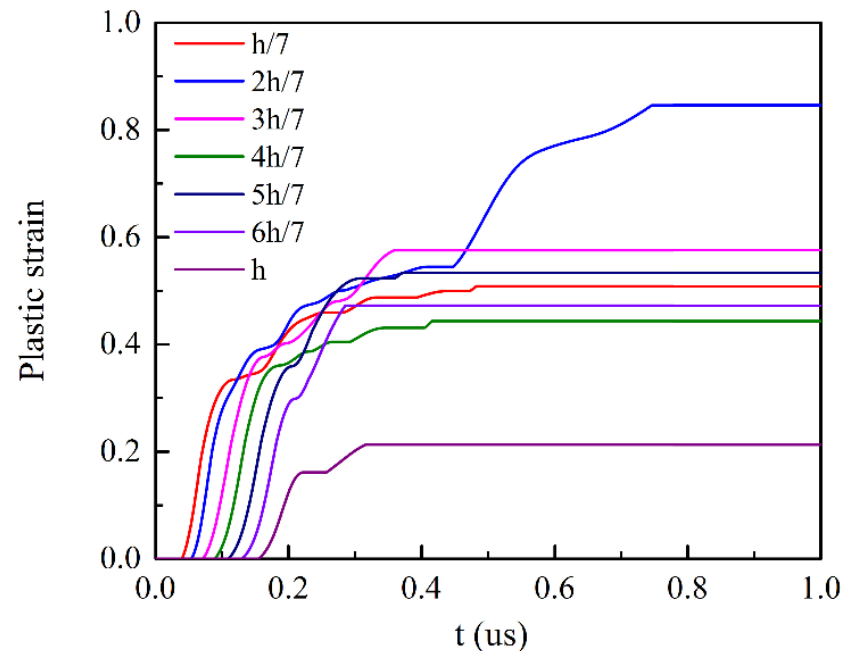

(c)

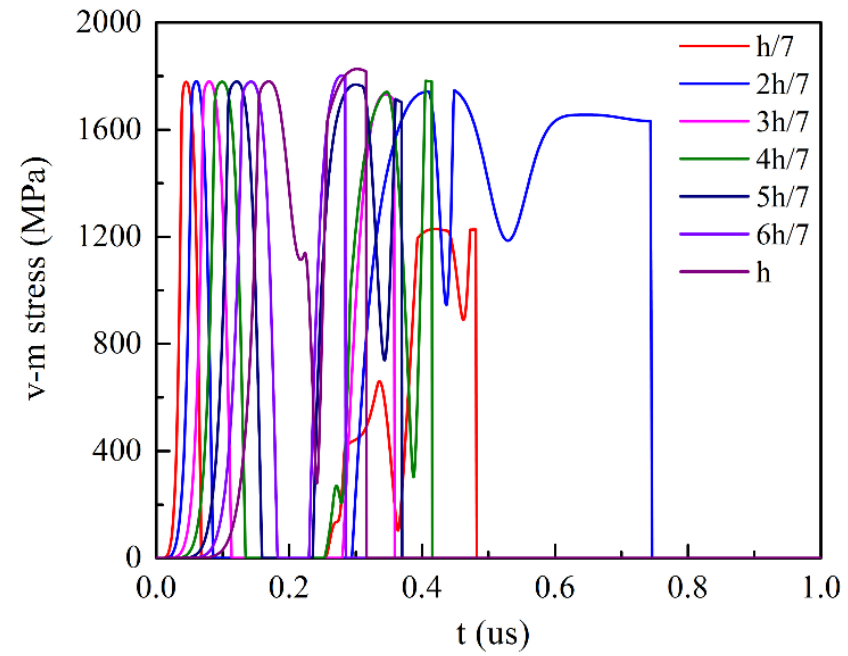

(e)

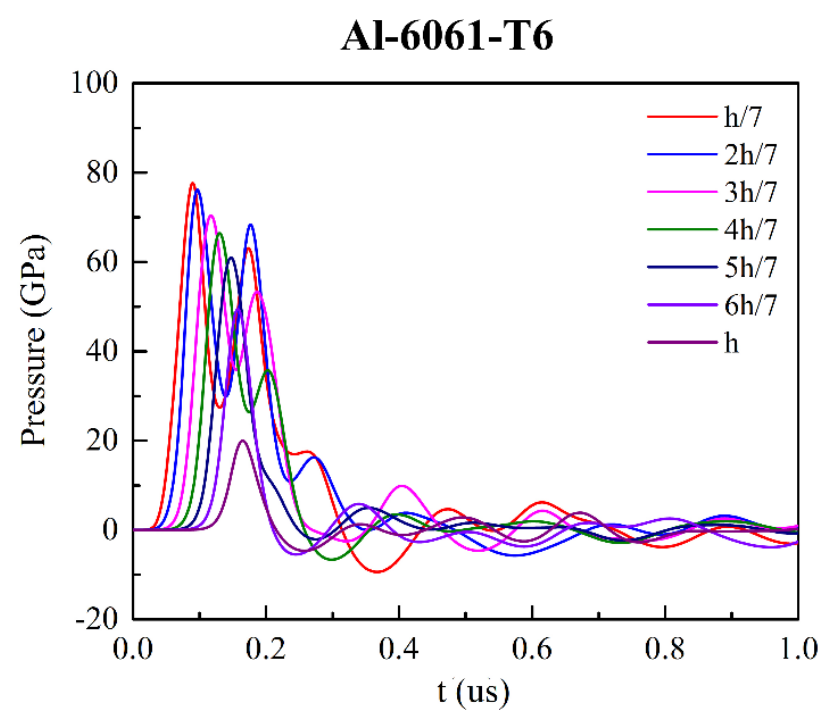

(b)

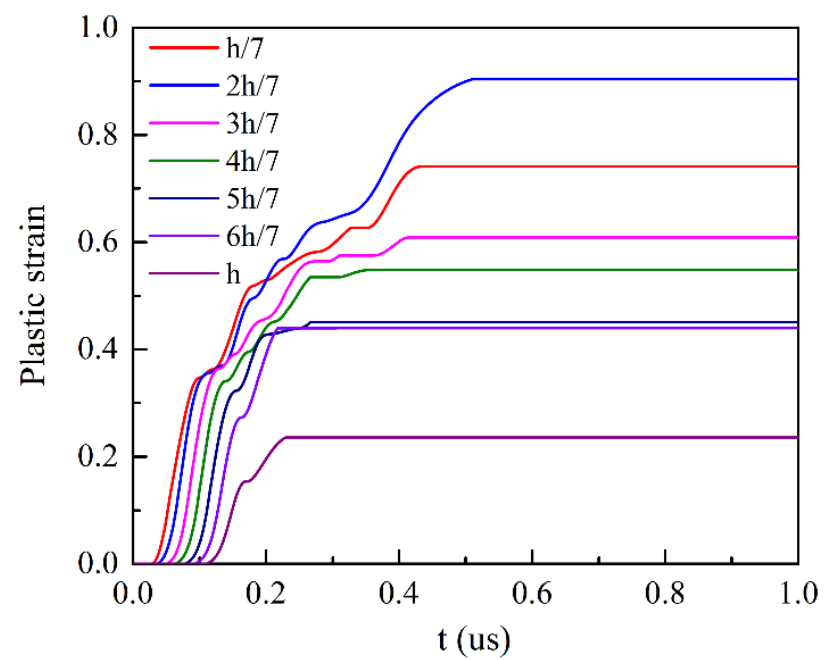

(d)

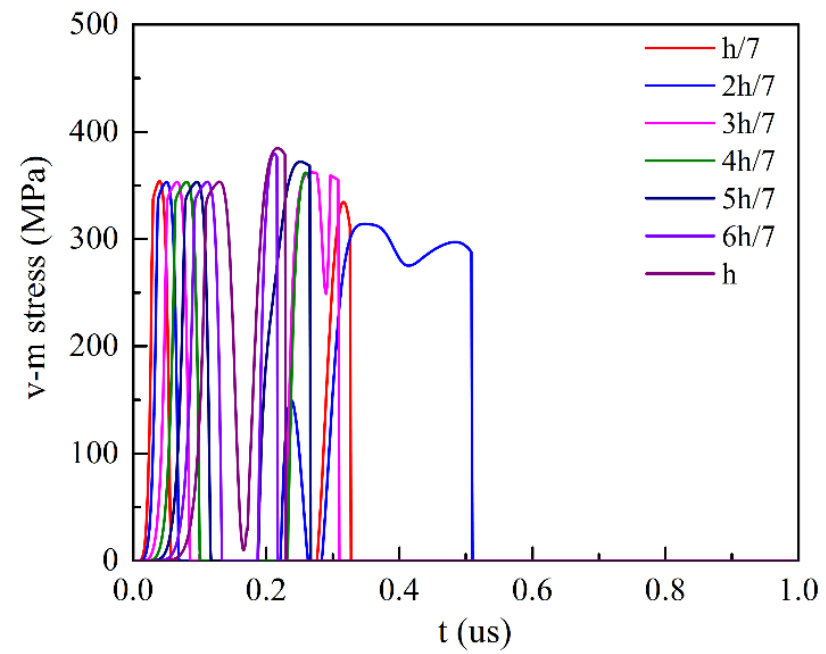

(f)

Fig. 5 The mechanical properties history of the sampling points along with the thickness of MGs and Al-6061-T6 bumpers, respectively. (a), (b) Pressure (c), (d) plastic strain and (e), (f) von-Mises stress. 


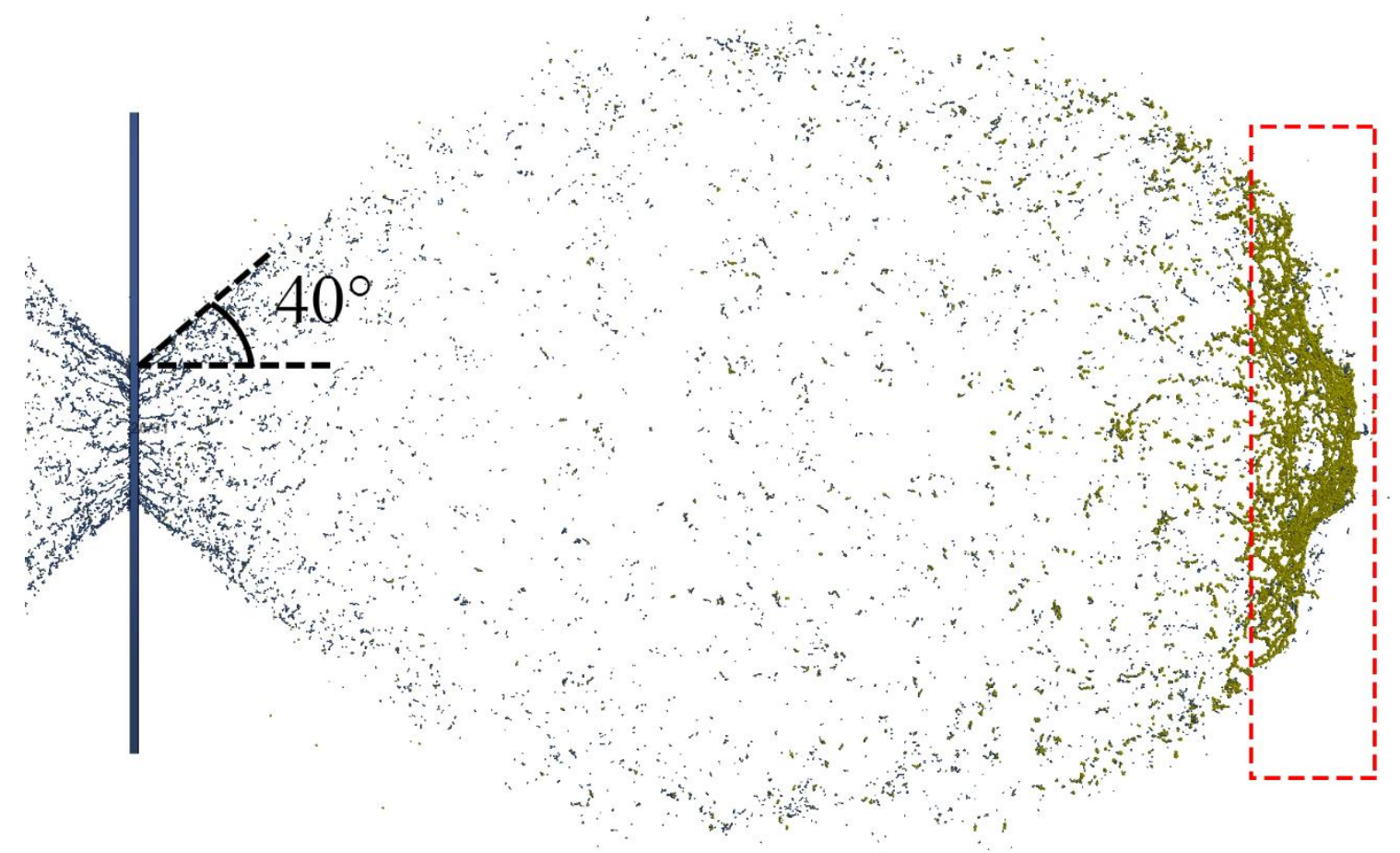

(a)

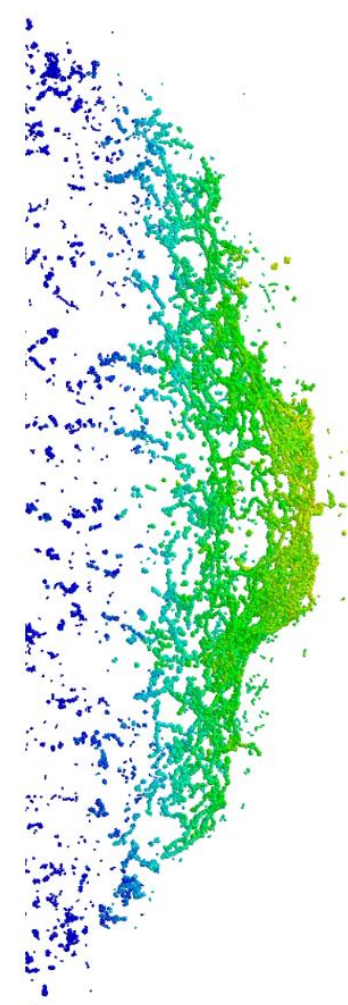

(b)

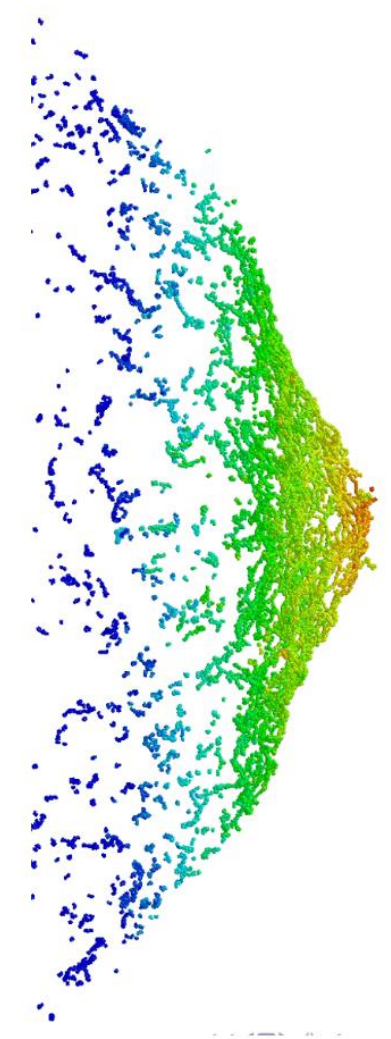

(c)

Fig. 6 (a) The morphology of debris cloud with a $0.5 \mathrm{~mm}$ thickness MGs bumper at 15 us (b) the velocity contours of the vanguard inside the red dashed box zone and (c) the vanguard of case 2 .

In actual working conditions, the bumper is usually designed to have a lower areal density with a better protective performance. Fig. 6a shows the morphology of the debris cloud with 0.5-mm-thickness Ti-MGs bumper and Al-6061-T6 bumper with the equal areal density. The mass 
of debris cloud of Ti-MGs bumper become concentrated on the vanguard rather dispersive. Moreover, the morphology features of debris cloud of thinner MGs bumper is much similar with the same areal density bumper made of Al-6061-T6. The maximum vanguard velocity of $\mathrm{Al} / \mathrm{Al}$ is still higher than the $\mathrm{Al} / \mathrm{MGs}$. However, the shape of the vanguard of $\mathrm{Al} / \mathrm{MGs}$ is more like a plane rather a sharpness which indicates that the MGs bumper makes the kinetic energy of debris cloud more evenly distributed than A1-6061-T6 bumper when the debris cloud impacts a rear plate. Furthermore, the dispersion angle with 0.5 -mm-thickness Ti-MGs bumper is about $40^{\circ}$ which is still larger than with Al-6061-T6 bumper. All evidence indicates that using the MGs as a bumper can absorb the more kinetic energy of the projectile and make the debris cloud more dispersed than Al-6061-T6.

\section{Conclusion}

The characteristic and mechanical response of MGs bumper under hypervelocity impacts condition were investigated by SPH/FEM methods in this paper and the following conclusions are drawn. Three cases of numerical simulation are calculated and the detailed mechanical responses of MGs are obtained. With the same thickness bumper, the peak values of pressure reach to $95 \mathrm{GPa}$ in the Ti-MGs bumper while in the Al-6061-T6 bumper, the peak value is only about 80GPa. Also, the ejection and dispersion angle of the debris cloud is larger than the Al-6061-T6 plate. Furthermore, the debris fragments are relatively uniformly distributed in the front part of the debris cloud.

Moreover, with the same areal density bumper, the calculation of the Ti-MGs bumper still has a better performance than the Al-6061-T6 bumper due to Ti-MGs having a much higher strength to density ratio. Besides, hypervelocity impacts can produce an extremely high temperature at the impact zone, and the viscosity of MGs will dramatically decreasing at glass transition temperature $\mathrm{T}_{\mathrm{g}}$ which is lower than the melting temperature of Al-6061-T6. That may be another reason that MGs bumper has a better performance against hypervelocity impact.

\section{Acknowledgments}

The authors would like to acknowledge support by the National Natural Science Foundation of China (Grant No. 51401028, No. 51271193, No. 11402277, No. 11790292) and the Strategic Priority Research Program of the Chinese Academy of Sciences (Grant No. XDB22040303) and also by the Innovation Program (237099000000170004).

\section{References}

[1] F.L. Whipple, Meteorites and space travel, Astron. J. 52 (1947) 131.

[2] M. Davidson, S. Roberts, G. Castro, R.P. Dillon, A. Kunz, H. Kozachkov, M.D. Demetriou, W.L. Johnson, S. Nutt and D.C. Hofmann, Investigating Amorphous Metal Composite Architectures as Spacecraft Shielding, Adv. Eng. Mater. 15 (2013) 27-33.

[3] X. Huang, Z. Ling, Z.D. Liu, H.S. Zhang and L.H. Dai, Amorphous alloy reinforced Whipple shield structure, Int. J. Impact Eng. 42 (2012) 1-10.

[4] L. Hamill, S. Roberts, M. Davidson, W.L. Johnson, S. Nutt and D.C. Hofmann, Hypervelocity Impact Phenomenon in Bulk Metallic Glasses and Composites, Adv. Eng. Mater. 16 (2014) 85-93.

[5] D.C. Hofmann, L. Hamill, E. Christiansen and S. Nutt, Hypervelocity Impact Testing of a Metallic Glass-Stuffed Whipple Shield, Adv. Eng. Mater. 17 (2015) 1313-1322.

[6] W. Zheng, Y.J. Huang, B.J. Pang and J. Shen, Hypervelocity impact on $\mathrm{Zr}_{51} \mathrm{Ti}_{5} \mathrm{Ni}_{10} \mathrm{Cu}_{25} \mathrm{Al}_{9}$ bulk metallic glass, Mater. Sci. Eng. A 529 (2011) 352-360.

[7] R.A. Gingold and J.J. Monaghan, Smoothed particle hydrodynamics: theory and application to non-spherical stars, Mon. Not. R. Astron. Soc. 181 (1977) 375-389. 
[8] L.D. Libersky and A.G. Petschek, Smooth particle hydrodynamics with strength of materials, in Advances in the free-Lagrange method including contributions on adaptive gridding and the smooth particle hydrodynamics method, Springer, 1991, pp. 248-257.

[9] L.B. Lucy, A numerical approach to the testing of the fission hypothesis, Astron. J. 82 (1977) 1013.

[10] G.R. Johnson, A constitutive model and data for materials subjected to large strains, high strain rates, and high temperatures, Proc. 7th Inf. Sympo. Ballistics, (1983) 541-547.

[11] G.R. Johnson and W.H. Cook, Fracture characteristics of three metals subjected to various strains, strain rates, temperatures and pressures, Engineering Fracture Mechanics, 21(1985) 31-48.

[12] F. Yuan, V. Prakash and J.J. Lewandowski, Spall strength of a zirconium-based bulk metallic glass under shock-induced compression-and-shear loading, Mech. Mater. 41 (2009) 886-897.

[13] F. Yuan, V. Prakash and J.J. Lewandowski, Spall strength and Hugoniot elastic limit of a zirconium-based bulk metallic glass under planar shock compression, J. Mater. Res. 22 (2011) 402-411.

[14] F. Xi, Y. Yu, C. Dai, Y. Zhang and L. Cai, Shock compression response of a Zr-based bulk metallic glass up to $110 \mathrm{GPa}$, J. Appl. Phys. 108 (2010) 083537.

[15] S.G. Ma, Z.M. Jiao, J.W. Qiao, H.J. Yang, Y. Zhang and Z.H. Wang, Strain rate effects on the dynamic mechanical properties of the $\mathrm{AlCrCuFeNi} 2$ high-entropy alloy, Mater. Sci. Eng. A 649 (2016) 35-38.

[16] J.W. Qiao, M.Y. Chu, L. Cheng, H.Y. Ye, H.J. Yang, S.G. Ma and Z.H. Wang, Plastic flows of in-situ metallic glass matrix composites upon dynamic loading, Mater. Lett. 119 (2014) 92-95.

[17] R.F. Wu, Z.M. Jiao, Y.S. Wang, Z. Wang, Z.H. Wang, S.G. Ma and J.W. Qiao, Excellent plasticity of a new Ti-based metallic glass matrix composite upon dynamic loading, Mater. Sci. Eng. A 677 (2016) 376-383.

[18] M.Y. Chu, Z.M. Jiao, Z.H. Wang, Y.S. Wang, J.H. Zhang, H.J. Yang and J.W. Qiao, Different deformation behaviors of two in-situ Ti-based metallic glass matrix composites upon quasi-static and dynamic compressions, Mater. Sci. Eng. A 639(2015) 717-723.

[19] J. Pan, Y.X. Wang and Y. Li, Ductile fracture in notched bulk metallic glasses, Acta Mater. 136 (2017) 126-133.

[20] W. Ma, H. Kou, J. Li, H. Chang and L. Zhou, Effect of strain rate on compressive behavior of Ti-based bulk metallic glass at room temperature, J. Alloys Compd. 472 (2009) 214-218.

[21] M.V. Silnikov, I.V. Guk, A.F. Nechunaev and N.N. Smirnov, Numerical simulation of hypervelocity impact problem for spacecraft shielding elements, Acta Astronaut. 150 (2018) 56-62.

[22] A.I.H. Committee, Properties and selection: nonferrous alloys and special-purpose materials, Asm Intl. Vol. 2, 1990.

[23] Y.C. Kim, W.T. Kim and D.H. Kim, A development of Ti-based bulk metallic glass, Mater. Sci. Eng. A 375 (2004) 127-135. 